\title{
Mechanically axially chiral catenanes and noncanonical chiral rotaxanes
}

John R. J. Maynard, ${ }^{+}$Peter Gallagher, ${ }^{+}$David Lozano, Patrick Butler and Stephen M. Goldup*

'These authors contributed equally

Chemistry, University of Southampton, Highfield, Southampton, SO17 1BJ (UK), *s.goldup@soton.ac.uk

\section{SUMMARY}

Chirality, the property of objects that are distinct from their own mirror image, is important in many scientific areas but particularly chemistry, where the appearance of molecular chirality because of rigid arrangements of atoms in space famously influences a molecule's biological properties. Less generally appreciated is that two molecular rings with chemically distinct faces combined like links in a chain results in a chiral structure even when the rings are achiral. To date, no enantiopure examples of such mechanically axially chiral catenanes has been reported. We re-examined the symmetry properties of the mechanically axially chiral motif and identified a straightforward route to such molecules from simple building blocks. We also identify that common representations of axially chiral catenanes obscure that a previously overlooked stereogenic unit arises when a ring is threaded onto a dumbbell-shaped molecule to generate a rotaxane. These insights allowed us to demonstrate the first stereoselective syntheses of an axially chiral catenane and a noncanonical axially chiral rotaxane motif. With methods to access these structures in hand, the process of exploring their properties and applications can now begin.

\section{INTRODUCTION}

The shape of a molecule is a major determinant of its function ${ }^{1}$. Thus, chemists have invested significant effort in developing methods to produce molecules with control over their stereochemistry, the formal term that refers to the factors that determine molecular shape ${ }^{2}$. A major part of this effort, which has now led to two Nobel prizes ${ }^{3,4}$, has focused on being able to selectively make molecules in one mirror image form because the non-superimposable mirror image forms (enantiomers) of chiral molecules are hard to separate using standard techniques. Although chirality is a whole-molecule property ${ }^{5}$, chemists often trace the appearance of molecular chirality back to one or more rigidly chiral arrangements of atoms in the structure. The most famous of these is the "stereogenic center" embodied by a tetrahedral carbon atom bonded to four different substituents, although stereogenic planes and axes are also found in important natural and synthetic structures ${ }^{2}$. Chiral molecules containing such classical covalent stereogenic units 
have been studied extensively. Less explored are chiral molecules whose stereochemistry arises absent any covalent stereogenic unit, such as Möbius ladders, ${ }^{6}$ molecular knots, ${ }^{7}$ and mechanically interlocked molecules ${ }^{8}$.

In 1961 Wasserman and Frisch identified that interlocked molecules called catenanes (two molecular rings joined like links in a chain) can display non-classical "mechanical" stereochemistry9; when both rings are oriented ( $C_{n h}$ symmetry) a catenane exists in two mirror image forms (Figure 1a). A decade later, Schill proposed that rotaxanes (a molecular ring trapped around a dumbbell-shaped molecule) composed of an oriented ring encircling an axle whose ends are distinct are also chiral (Fig. 1a) ${ }^{10}$. In both cases, the subcomponents that make up the interlocked structure are not themselves chiral, which is readily emphasized using commonly employed schematic representations that focus on the symmetry properties of the components (Fig. 1a). These representations also make clear that such topologically chiral catenanes and mechanically planar chiral rotaxanes are related notionally through ring opening. Although such molecules were initially challenging to make as single enantiomers ${ }^{11,12,13,14,15}$, recent efforts have allowed them to be accessed in good enantiopurity using standard synthetic approaches ${ }^{16,17,18,19,20,21}$.

In 2002 Puddephat and co-workers reported a chiral catenane composed of two achiral rings with distinct faces $\left(\mathrm{C}_{\mathrm{nv}}\right)$ (Fig. 1b). However, unlike the example of topologically chiral catenanes, the notional process of ring opening and stoppering of the minimal representation of such axially chiral catenanes does not result in a chiral rotaxane (Fig. 1b). A small number of racemic axially chiral catenanes have been reported $^{22,23}$, but no enantiopure examples where the mechanical bond provides the sole source of stereochemistry have been disclosed to date ${ }^{24}$. We re-examined the mechanical axial stereogenic unit of catenanes with a focus on not just the symmetry of the components but how this arises structurally. This led us not only to an efficient approach to enantiopure axially chiral catenanes but also to recognize and synthesize a noncanonical class of mechanically axially chiral rotaxanes that had previously been overlooked. 
Figure 1. Schematic depictions of the mechanical stereogenic units of chiral catenanes and rotaxanes (stereolabels are arbitrary). (a) The mechanical topological and planar chiral stereogenic units of catenanes and rotaxanes are related by a notional ring opening process. (b) The minimal schematic representation of a mechanically axially chiral catenane suggests that there is no analogous axially chiral rotaxane. (c) Semi-structural representations of axially chiral catenanes reveal that such molecules can display co-conformational covalent chirality alongside the fixed mechanical stereogenic unit. (d) The semi-structural representation reveals that rotaxanes display a related but previously unrecognized form of stereochemistry.

a.

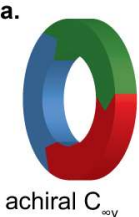

macrocycle
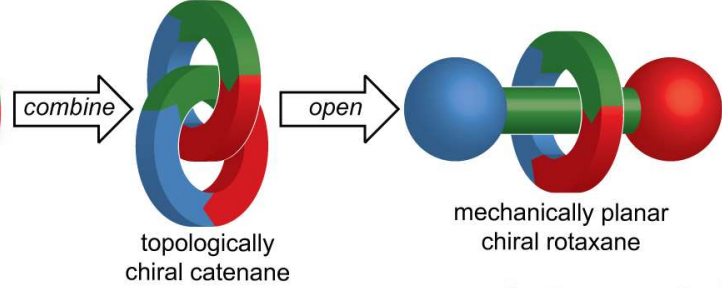

b.
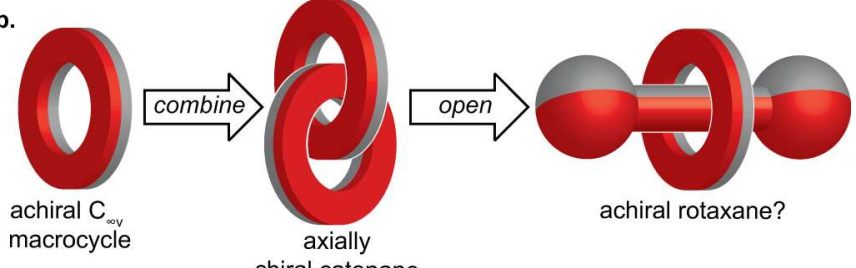

c.

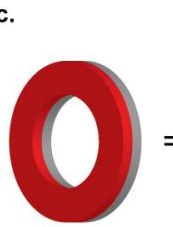

chiral catenane chiral rotaxane macrocycle

chiral catenan

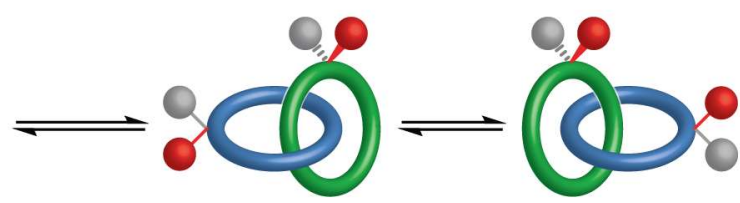

$\left(S_{\mathrm{ma}}, R_{\mathrm{coc}-\mathrm{c}}\right)$-III

dynamic co-conformational covalent chiral /mechanically axially chiral epimers

mechanically axially chiral enantiomers

d.
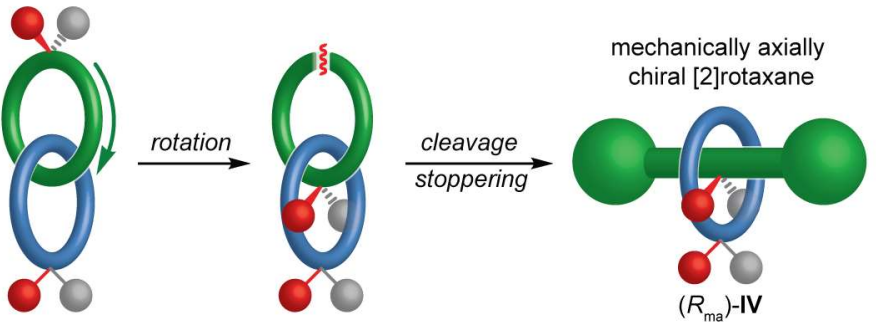

dynamically co-conformational covalent chiral / mechanicallyaxially chiral diastereomer

\section{RESULTS AND DISCUSSION}

Insights from semi-structural cartoons. The minimal cartoon representation of a mechanically axially chiral catenane (Fig. 1b) does not specify how the facial dissymmetry of the macrocycles arises. The most obvious way this can be achieved is by including a prochiral unit in both rings (I, Fig. 1 c $)^{22,23,24,}$. Indeed, it is hard to avoid the appearance of prochirality ${ }^{25}$, whether that arises due to a center (shown), plane or axis, in the structure of mechanically axially chiral catenanes. Furthermore, although there is always a highly symmetrical arrangement of rings available (II) that only expresses mechanical axial stereochemistry, if either ring is displaced from this arrangement the resulting structure contains both a mechanically axially chiral stereogenic unit and a co-conformational covalent stereogenic unit (III). These lower symmetry arrangements exist as pairs of co-conformational diastereomers.

Strikingly, whereas the minimal cartoon representation of a mechanically axially chiral catenane suggests there can be no rotaxane equivalent of this stereogenic unit (Fig. 1b), the semi-structural 
representation reveals that the notional ring opening process gives rise to a chiral rotaxane (Fig. 1d); even when the ring encircles the prochiral unit of the axle (IV) there is no representation that is achiral. Thus, we see that rotaxanes can display a previously unremarked upon mechanically axially chiral stereogenic unit that displays similar co-conformational diastereoisomerism (V).

Having recognized that co-conformational diastereoisomerism is a fundamental property of mechanically axially chiral catenanes and rotaxanes, we proposed that a co-conformational stereogenic unit could act as a temporary source of chiral information in their synthesis (Fig. 2). By forming a mechanical bond selectively on one side of a prochiral unit (route a) and designing the structure such that co-conformational exchange is blocked, the mechanically axially chiral catenane product would be formed as a pair of separable diastereomers with identical co-conformational configuration (here $R_{\text {co-c }}$ ) but opposite mechanical axial configuration $\left(R_{\mathrm{ma}}\right.$ or $\left.S_{\mathrm{ma}}\right)$. Alternatively, installing a facially symmetrical ring on one side of a prochiral center would give rise to a single co-conformational enantiomer (route b). Subsequent desymmetrisation of the faces of the ring would give rise to the same pair of diastereomers. Removal of the groups preventing co-conformational motion would give mechanically axially chiral enantiomers in which the mechanical bond provides the sole fixed source of stereochemistry. An advantage of this co-conformational chiral auxiliary approaches is that co-conformational enantiomers can be made using chiral pool starting materials by choosing where the mechanical bond is formed $11,26,27,28$.

Figure 2. Proposed co-conformational auxiliary approach for the synthesis of axially chiral catenanes. If the prochiral substituents and blocking groups are large enough to prevent co-conformational isomerism, the diastereomers can be separated and then converted into enantiomeric axially chiral catenanes.

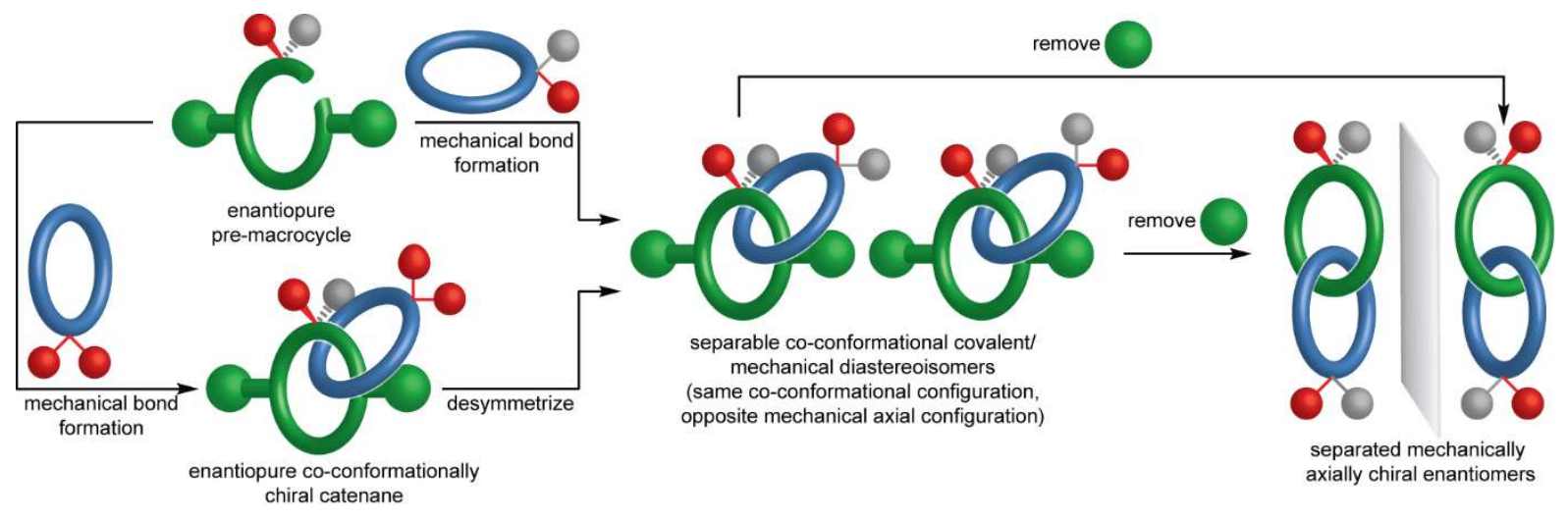


Proof-of-concept synthesis of an axially chiral catenane and rotaxane. $(R)$-Serine was elaborated to pre-macrocycle (R)-1 (Supplementary section 2) (Fig. 3a). Macrocycle 2, which contains a prochiral sulfoxide, was readily synthesized (Supplementary section 3) using a Ni-mediated macrocyclization protocol $^{29}$. Catenane formation was achieved by reacting $(R)-1$ with macrocycle 2 under active template ${ }^{30}$ Cu-mediated alkyne-azide cycloaddition (AT-CuAAC) $)^{31}$ conditions $^{32}$ to give separable diastereomers 3 in which co-conformational motion is prevented by bulky ester and $\mathrm{N}$-Boc groups (route a). Catenanes 3 could also be accessed by reaction of $(R)-1$ with macrocycle 4 to give $\left(R_{\text {co-c }}\right)-5$ followed oxidation to give catenanes 3 (route b). Both routes proceeded with appreciable but opposite stereoselectivity. Single crystal x-ray diffraction (SCXRD) analysis of the major product of of rac-1 and 2 allowed the major stereoisomer produced in routes $\mathrm{a}$ and $\mathrm{b}$ to be assigned. Conversion of diastereomers 3 to structures in which the mechanically axially chiral stereogenic unit is the only fixed source of stereochemistry can be achieved by removing the Boc group (Fig. 2b) or reducing the esters (Supplementary sections 5 and 6 respectively). Accordingly, removal of the Boc group from $\left(R_{\mathrm{ma}}, R_{\mathrm{co-c}}\right)-3$ or $\left(S_{\mathrm{ma}}, R_{\mathrm{co}-\mathrm{c}}\right)-\mathbf{3}$ gave $\left(R_{\mathrm{ma}}\right)-6$ ( $>99 \%$ e.e.) and ( $\left.S_{\mathrm{ma}}\right)-6$ (>99\% e.e.) respectively (Fig. 3e). The enantiomeric nature of these structures is supported by circular dichroism (CD) analysis (Fig. 3f). The solid-state structure of rac-6 (Fig. 3d) contains both co-conformational diastereomers with the $\left(R^{*}{ }_{\text {ma }}-R^{*}{ }_{\text {co-c }}\right)$ co-conformation observed to dominate $(\sim 80$ : 20). 
Figure 3. Synthesis and analysis of enantiopure axially chiral catenane 6. (a) Synthesis and separation of catenane diastereomers 3 from $(R)-1$ by route $a$ or route b (Fig. 2) with opposite diastereoselectivity. Reagents and conditions: i. [Cu(MeCN) $)_{4} \mathrm{PF}_{6}, \mathrm{~N}^{\prime} \mathrm{Pr}_{2} \mathrm{Et}, \mathrm{CH}_{2} \mathrm{Cl}_{2}$, rt, 16 h; ii. IBX, $\mathrm{NEt}_{4} \mathrm{Br}, \mathrm{CHCl}_{3}-\mathrm{H}_{2} \mathrm{O}(99$ : 1), rt, $16 \mathrm{~h}$. (b) Conversion of catenane 3 to enantiomeric catenanes 6 . Reagents and conditions: $\mathrm{CF}_{3} \mathrm{CO}_{2} \mathrm{H}, \mathrm{CH}_{2} \mathrm{Cl}_{2}, 0{ }^{\circ} \mathrm{C}, 1 \mathrm{~h}$. (c) The solid-state structure of rac- $\left(S_{\mathrm{ma}}, R_{\mathrm{co-c}}\right)-4$ allowed the major products of routes $\mathrm{a}$ and $\mathrm{b}$ to be assigned. (d) The solid-state structure of $(\mathrm{rac})-\mathbf{6}$ contains $\left(S_{\mathrm{ma}}, R_{\mathrm{co}-\mathrm{c}}\right)-\mathbf{6}$ as the major co-conformational diastereomer. Analysis of the enantiomers of catenane 6 by (e) CD and (f) HPLC confirmed their enantiopurity and chiral nature. IBX $=2$-iodoxybenzoic acid.

a.

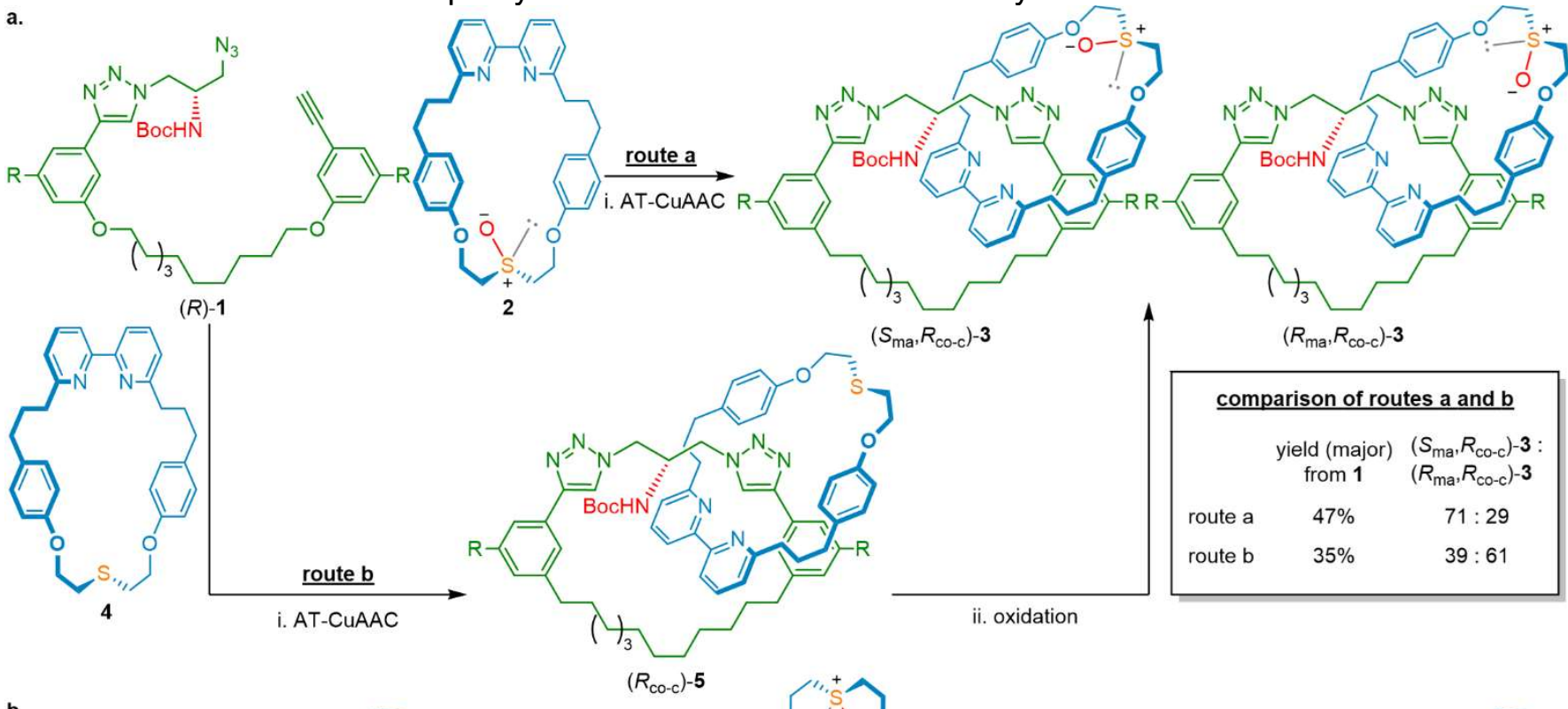

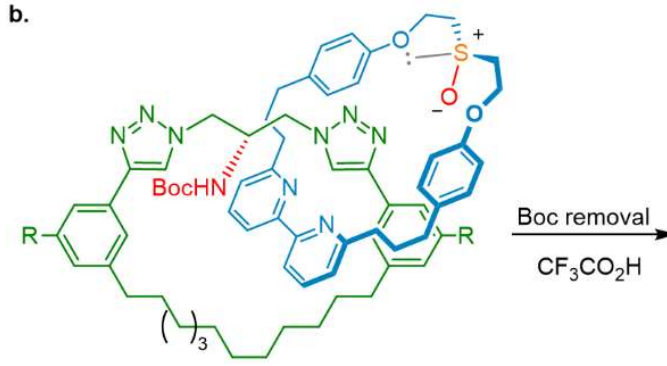

$\left(R_{\mathrm{ma}}, R_{\mathrm{co-c}}\right)-3$

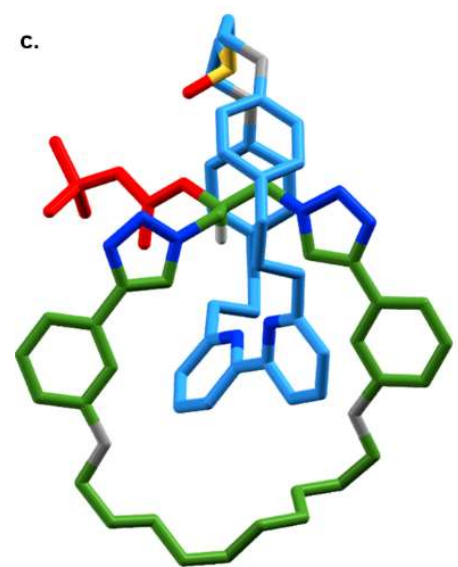

d.

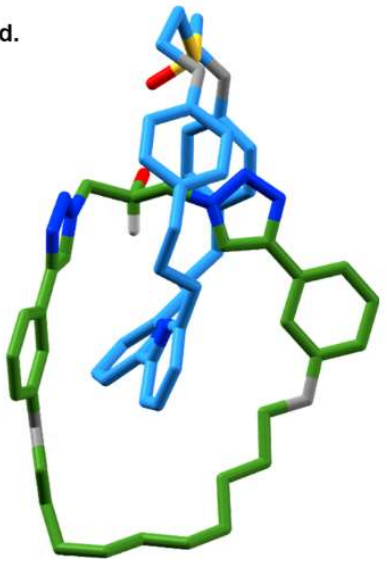

e.

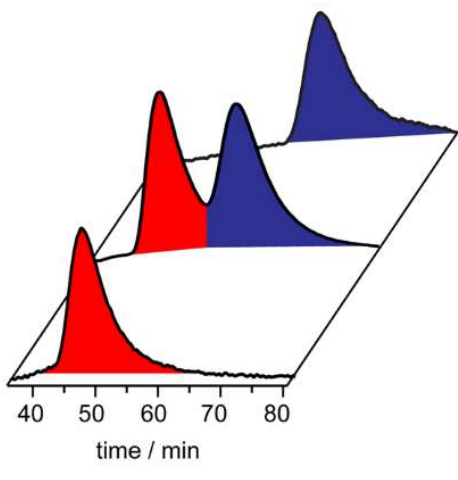

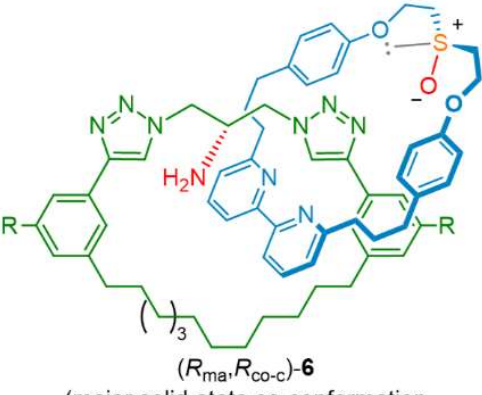

(major solid-state co-conformation

f.

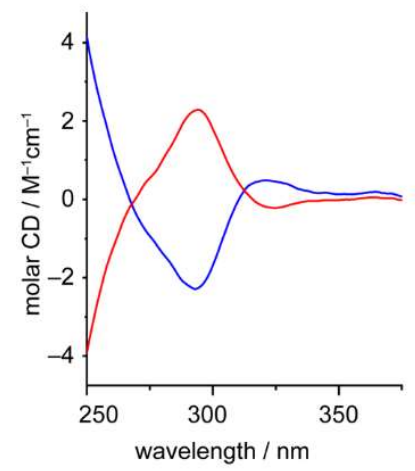

The same strategy was used to synthesize mechanically axially chiral rotaxane 11 (Fig. 3). Serinederived azide (R)-7 (Supplementary section 7), alkyne 8 and macrocycle 2 were reacted under AT-CuAAC conditions $^{33}$ to give rotaxane diastereomers 9 (route a). Rotaxanes 9 could also be accessed by reaction of $(R)-7,8$ and macrocycle 4 followed by oxidation (route b). SCXRD analysis of the major isomer obtained 
using route $\mathrm{b}$ with $(R)-7$ (Fig. $3 \mathrm{~b}$ ) allowed the major products of routes a and $\mathrm{b}$ to be assigned. Removal of the Boc group from separated samples of $\left(R_{\mathrm{ma}}, R_{\mathrm{co}-\mathrm{c}}\right)-\mathbf{9}$ and $\left(S_{\mathrm{ma}}, R_{\mathrm{co-c}}\right)-\mathbf{9}$ gave $\left(R_{\mathrm{ma}}\right)-10$ and $\left(S_{\mathrm{ma}}\right)-10$ respectively in excellent enantiopurity (>99\% e.e., Fig. $4 \mathrm{c}) .\left(R_{\mathrm{ma}}\right)-10$ and $\left(S_{\mathrm{ma}}\right)-10$ produce mirror-image CD spectra (Fig. 3d) emphasizing the chiral nature of the mechanical axial stereogenic unit.

Figure 4. Synthesis of mechanically axially chiral rotaxane 10. (a) Synthesis of diastereomeric mechanically axially chiral rotaxanes 9 by route a or b gives separable rotaxanes 9 that are converted to 10 by removal of the Boc group. Reagents and conditions: i. macrocycle 2 (route a) or macrocycle 4 (route b), [Cu(MeCN) $\left.{ }_{4}\right] \mathrm{PF}_{6}, \mathrm{NiPr}_{2} \mathrm{Et}_{1} \mathrm{CH}_{2} \mathrm{Cl}_{2}, \mathrm{rt}$, 16 h; ii. IBX, NEt 4 Br, $\mathrm{CHCl}_{3} \mathrm{H}_{2} \mathrm{O}$ (99 : 1), rt, 16 h; iii. $\mathrm{CF}_{3} \mathrm{CO}_{2} \mathrm{H}, \mathrm{CH}_{2} \mathrm{Cl}_{2}$, rt, 16 h. (d) SCXRD analysis of ( $\left.S_{\text {ma }}, R_{\text {co-c) }}\right)-9$ allowed the major products of routes $a$ and $b$ to assigned. Analysis of the enantiomers of rotaxane 9 by (c) CD and (d) HPLC confirmed their enantiopurity and chiral nature. IBX $=2$-iodoxybenzoic acid.
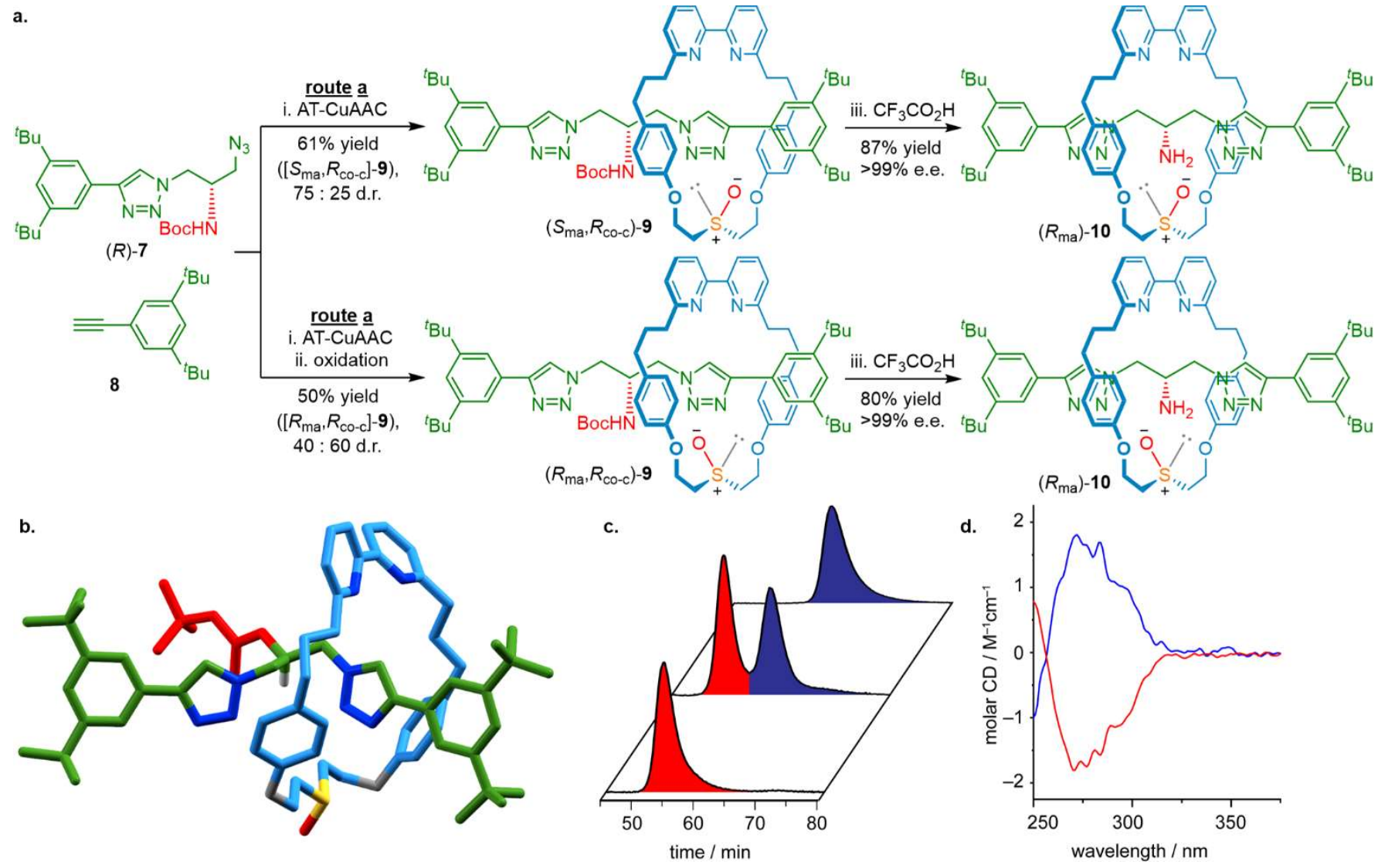

Stereochemical assignment and properties of the mechanically axially chiral stereogenic unit. The assignment of the mechanically axially chiral stereogenic unit relies on identifying the highest priority faces of each ring, which is only possible if the relative orientation of the prochiral units is specified. Building on a proposal by Stoddart and Bruns, we suggest that in the case of catenanes the in-plane substituents of the prochiral moieties be oriented so they "point" towards one another (Fig. 5a). Conversely, in the equivalent rotaxane, we suggest they be oriented to point in the same direction (Fig. 5b). This counterintuitive proposal, which is subject to approval by the community, is designed to ensure that a 
mechanically axially chiral rotaxane generated from an axially chiral catenane by ring opening would retain the same stereolabel.

Finally, we considered the nature of the stereochemistry in catenanes where one or both prochiral units are replaced with covalent stereocentres as these represent logical precursors to axially chiral targets if these centers were subsequently symmetrized and recently there has been a suggestion that the latter class might contain both mechanical axial and topological stereogenic units ${ }^{34}$. In the former (Fig. 5c), ligand permutation analysis reveals two diastereomers (shown) and their enantiomers (i.e. four stereoisomers), consistent with one covalent centre and one mechanical axial stereogenic unit. In the latter case, ligand permutation reveals four diasteromers (shown) and their enantiomers (eight stereoisomers), consistent with two covalent and one mechanical stereogenic unit. However, the nature of the mechanical stereochemistry is ambiguous as each diastereomer can be assigned using either a mechanical axial or a mechanical topological stereodescriptor, only one of which is required to specify the structure. This suggests that it would be incorrect to describe such catenanes as simultaneously topologically and mechanically axially chiral - one of the stereolabels would be redundant. This analysis may appear philosophical in nature but has implications for the synthesis of chiral catenanes. If a single diastereomer of such a catenane could be isolated, it could be converted to an axially chiral catenane by selective symmetrization of the in-plane substituents, or a topologically chiral catenane by symmetrization of the out of plane substituents (Fig. 5e). This analysis further highlights that how a stereogenic unit is conceptualized can guide the development of new methodologies. 
Figure 5. Assignment and further analysis of the mechanical axial stereogenic unit. Methods to assign the stereogenic units of mechanically axially chiral (a) catenanes and (b) rotaxanes by specifying the relative orientation of prochiral moieties. (c) The two diastereomers identified in catenanes containing one prochiral and one fixed covalent stereogenic center. (d) The four diastereomers identified in catenanes containing a covalent stereogenic center in both rings whose structures can be specified using either a mechanical topological or axial stereodescriptor.

(e) Selective symmetrization of the in-plane or out of plane substituents of one diastereomers of (d) gives a topologically or axially chiral catenane respectively.
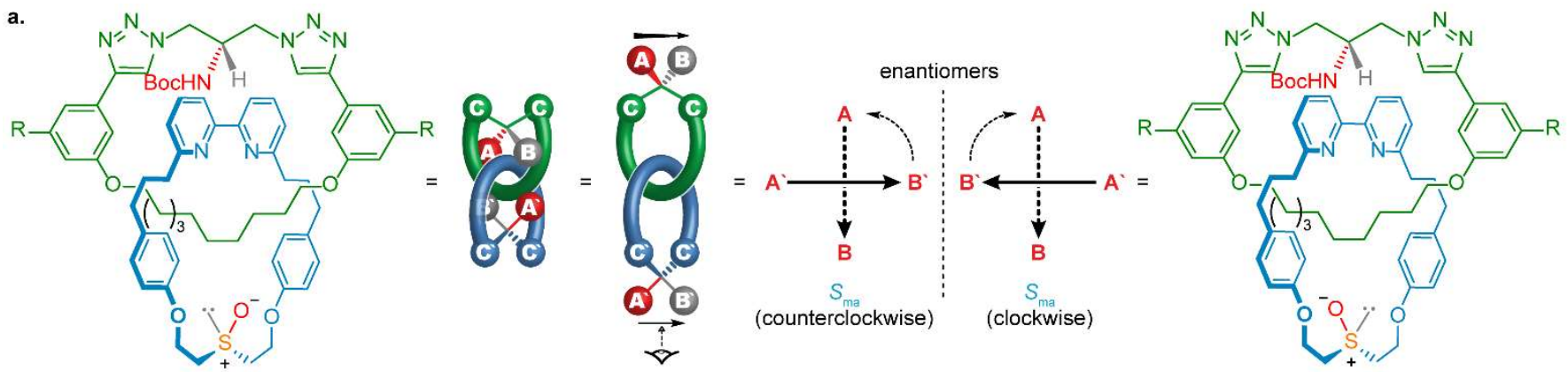

b.
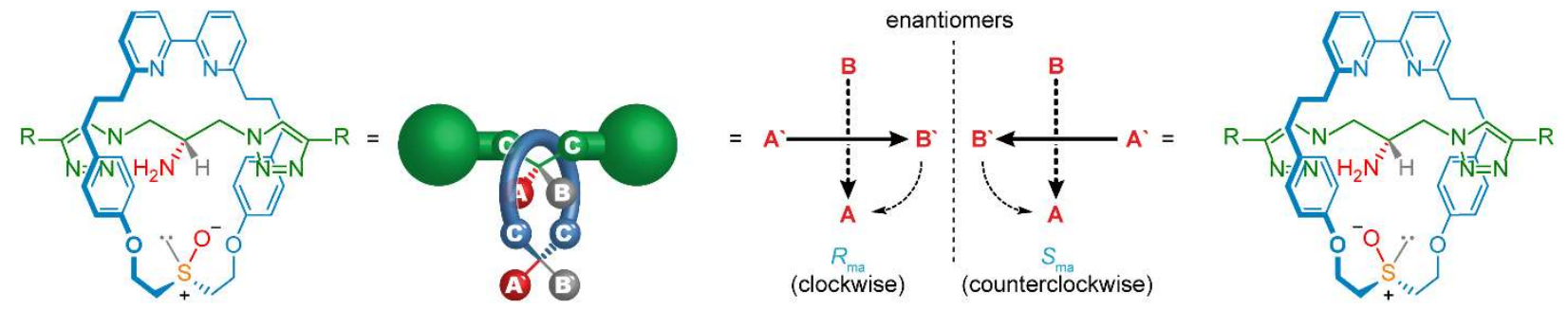

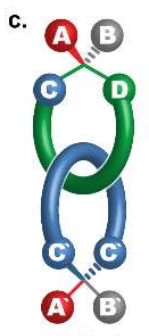

$\left(R, R_{\mathrm{ma}}\right)$

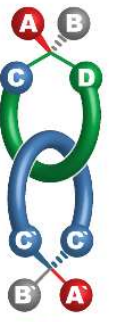

$\left(R, S_{\mathrm{ma}}\right)$ d.

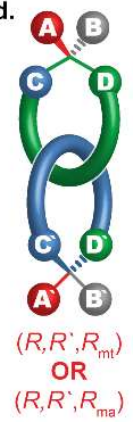

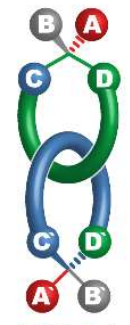

$\left(S, R, R_{\mathrm{mt}}\right)$ OR
$R, R^{\prime}, S$

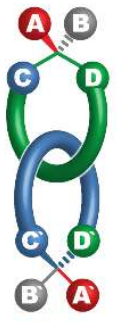

$\left(R, S^{\prime}, R_{\mathrm{mt}}\right)$ OR $\left(R, S^{\prime}, S_{\mathrm{ma}}\right)$

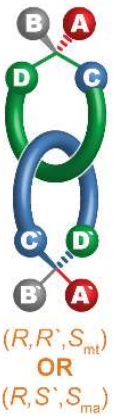

e.

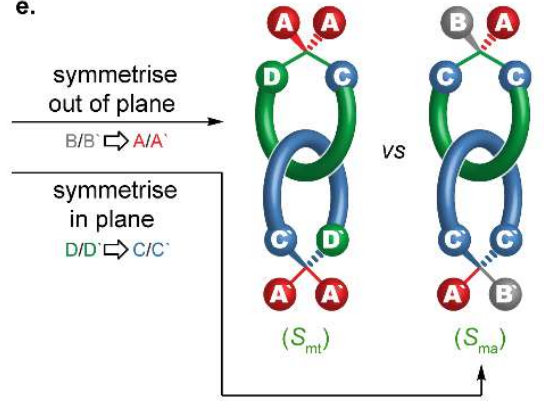

\section{CONCLUSIONS}

Semi-structural analysis allowed us to identify an efficient co-conformational auxiliary approach to mechanically axially chiral catenanes and revealed a previously overlooked axially chiral stereogenic unit in rotaxanes. The latter is a rare example of a "new" source of stereoisomerism, as opposed to a new pathway of isomer exchange $\mathrm{e}^{35,36}$ or an overlooked opportunity for atropisomerism ${ }^{37}$, as have recently been reported. However, it is so closely related to that of catenanes it is perhaps important consider how it was overlooked for so long which may be at least in part due to the use of schematic cartoons that focus on symmetry without reference to the chemical moieties and can obscure important chemical information. Furthermore, it appears sensible to ask how the stereoisomerism of rotaxanes and catenanes could be systematized and unified rather than treated as separate as they are typically ${ }^{38}$. The same semi-structural 
approach leads to the surprising conclusion that catenanes based on rings containing a single stereogenic center can be described as either mechanically topologically or axially chiral but that only one such stereodescriptor is required to specify their structure, an observation with implications for future syntheses. Given the increasing interest in applications of chiral interlocked molecules $39,40,41,42,43,28$ including examples based on mechanically chiral systems ${ }^{44,45,46}$, as well as other exotic or hard to access mechanical stereogenic units ${ }^{47,48,49,50,51}$, we anticipate these results will spur progress in the development of functional mechanically chiral systems ${ }^{52}$.

\section{ACKNOWLEDGEMENTS}

SMG thanks the ERC (Agreement no. 724987) and the Royal Society for a Wolfson Research Fellowship (RSWFIFT\180010). PB thanks the University of Southampton for a Presidential Scholarship.

\section{AUTHOR CONTRIBUTIONS}

JRJM and SMG developed the co-conformational auxiliary concept. JRJM synthesized $\mathbf{3}$ and $\mathbf{5}$ and collected SCXRD diffraction data for a reduced product of catenane 5. PRG synthesized 10 and 11. DL optimized the synthesis and purification of $\mathbf{3}, \mathbf{5}$, synthesized $\mathbf{6}$ and determined the stereochemistry of catenanes 3. PB collected the X-ray diffraction data of 3, 6 and 10 and fully refined all SCXRD data. DL and PRG managed the preparation of the Supporting Information. SMG directed the research. All authors contributed to the analysis of the results and the writing of the manuscript.

\section{COMPETING INTERESTS STATEMENT}

The authors declare no competing interests.

(1) Diaz, D. B. et al. Illuminating the dark conformational space of macrocycles using dominant rotors. Nat Chem 13, 218-225 (2021).

(2) Eliel, E. L., Wilen, S. H. \& Mander, L. N. Stereochemistry of Organic Compounds. (John Wiley and Sons, Inc., 1994).

(3) The Nobel Prize in Chemistry 2001 - NobelPrize.org, accessed 9/11/2021.

(4) The Nobel Prize in Chemistry 2021 - NobelPrize.org, accessed 9/11/2021.

(5) Mislow, K. \& Siegel, J. Stereoisomerism and Local Chirality. Journal of the American Chemical Society 106, 3319-3328 (1984). 
(6) Herges, R. Topology in chemistry: designing Mobius molecules. Chem Rev 106, 4820-4842 (2006).

(7) Fielden, S. D. P., Leigh, D. A. \& Woltering, S. L. Molecular Knots. Angew. Chem. Int. Ed. 56, 1116611194 (2017).

(8) Jamieson, E. M. G., Modicom, F. \& Goldup, S. M. Chirality in rotaxanes and catenanes. Chem Soc Rev 47, 5266-5311 (2018).

(9) Frisch, H. L. \& Wasserman, E. Chemical Topology. J. Am. Chem. Soc. 83, 3789-3795 (1961).

(10) Schill, G. Catenanes, Rotaxanes and Knots. (Academic Press, 1971).

(11) Maynard, J. R. J. \& Goldup, S. M. Strategies for the Synthesis of Enantiopure Mechanically Chiral Molecules. Chem 6, 1914-1932 (2020).

(12) Chambron, J. C., Dietrich-Buchecker, C., Rapenne, G. \& Sauvage, J. P. Resolution of topologically chiral molecular objects. Chirality 10, 125-133 (1998).

(13) Yamamoto, C., Okamoto, Y., Schmidt, T., Jager, R. \& Vogtle, F. Enantiomeric resolution of cycloenantiomeric rotaxane, topologically chiral catenane, and pretzel-shaped molecules: Observation of pronounced circular dichroism. J. Am. Chem. Soc. 119, 10547-10548 (1997).

(14) Hirose, K. et al. The Asymmetry is Derived from Mechanical Interlocking of Achiral Axle and Achiral Ring Components -Syntheses and Properties of Optically Pure [2]Rotaxanes-. Symmetry 10, 20 (2018).

(15) Gaedke, M. et al. Chiroptical inversion of a planar chiral redox-switchable rotaxane. Chem. Sci. 10, 10003-10009 (2019).

(16) Imayoshi, A. et al. Enantioselective preparation of mechanically planar chiral rotaxanes by kinetic resolution strategy. Nat. Commun. 12, 404 (2021).

(17) Tian, C., Fielden, S. D. P., Perez-Saavedra, B., Vitorica-Yrezabal, I. J. \& Leigh, D. A. Single-Step Enantioselective Synthesis of Mechanically Planar Chiral [2]Rotaxanes Using a Chiral Leaving Group Strategy. J. Am. Chem. Soc. 142, 9803-9808 (2020).

(18) Bordoli, R. J. \& Goldup, S. M. An efficient approach to mechanically planar chiral rotaxanes. J Am Chem Soc 136, 4817-4820 (2014).

(19) Jinks, M. A. et al. Stereoselective Synthesis of Mechanically Planar Chiral Rotaxanes. Angew. Chem. Int. Ed. 57, 14806-14810 (2018).

(20) Nat Chem!

(21) Denis, M., Lewis, J. E. M., Modicom, F. \& Goldup, S. M. An Auxiliary Approach for the Stereoselective Synthesis of Topologically Chiral Catenanes. Chem 5, 1512-1520 (2019).

(22) McArdle, C. P., Vittal, J. J. \& Puddephatt, R. J. Molecular Topology: Easy Self-Assembly of an Organometallic Doubly Braided [2]Catenane. Angew Chem Int Ed Engl 39, 3819-3822 (2000).

(23) McArdle, C. P., Van, S., Jennings, M. C. \& Puddephatt, R. J. Gold(I) macrocycles and topologically chiral [2]catenanes. J Am Chem Soc 124, 3959-3965 (2002).

(24) Theil, A., Mauve, C., Adeline, M.-T., Marinetti, A. \& Sauvage, J.-P. in Angewandte Chemie International Edition Vol. 45 2104-2107 (2006). 
(25) IUPAC. Compendium of Chemical Terminology, 2nd ed. (the "Gold Book"). Compiled by A. D. McNaught and A. Wilkinson. Blackwell Scientific Publications, Oxford (1997). Online version (2019-) created by S. J. Chalk. ISBN 0-9678550-9-8. https://doi.org/10.1351/goldbook.

(26) Alvarez-Perez, M., Goldup, S. M., Leigh, D. A. \& Slawin, A. M. A chemically-driven molecular information ratchet. J Am Chem Soc 130, 1836-1838 (2008).

(27) Carlone, A., Goldup, S. M., Lebrasseur, N., Leigh, D. A. \& Wilson, A. A three-compartment chemically-driven molecular information ratchet. J Am Chem Soc 134, 8321-8323 (2012).

(28) Cakmak, Y., Erbas-Cakmak, S. \& Leigh, D. A. Asymmetric Catalysis with a Mechanically Point-Chiral Rotaxane. J Am Chem Soc 138, 1749-1751 (2016).

(29) Lewis, J. E. M. et al. High yielding synthesis of 2,2'-bipyridine macrocycles, versatile intermediates in the synthesis of rotaxanes. Chemical Science 7, 3154-3161 (2016).

(30) Denis, M. \& Goldup, S. M. The active template approach to interlocked molecules. Nat Rev Chem 1, 0061 (2017).

(31) Aucagne, V., Hanni, K. D., Leigh, D. A., Lusby, P. J. \& Walker, D. B. Catalytic "click" rotaxanes: a substoichiometric metal-template pathway to mechanically interlocked architectures. J Am Chem Soc 128, 2186-2187 (2006).

(32) Lewis, J. E. M., Modicom, F. \& Goldup, S. M. Efficient Multicomponent Active Template Synthesis of Catenanes. J Am Chem Soc 140, 4787-4791 (2018).

(33) Lahlali, H., Jobe, K., Watkinson, M. \& Goldup, S. M. Macrocycle size matters: "small" functionalized rotaxanes in excellent yield using the CuAAC active template approach. Angew Chem Int Ed Engl 50, 4151-4155 (2011).

(34) Schroder, H. V., Zhang, Y. \& Link, A. J. Dynamic covalent self-assembly of mechanically interlocked molecules solely made from peptides. Nat. Chem. (2021).

(35) Canfield, P. J. et al. A new fundamental type of conformational isomerism. Nat Chem 10, 615-624 (2018). 
(36) Canfield, P. J., Govenlock, L. J., Reimers, J. \& Crossley, M. J. Recent Advances in Stereochemistry Reveal Classification Shortcomings. ChemRxiv (2020). doi:10.26434/chemrxiv.12488525.v1 This content is a preprint and has not been peer-reviewed.

(37) Reisberg, S. H. et al. Total synthesis reveals atypical atropisomerism in a small-molecule natural product, tryptorubin A. Science 367, 458-463 (2020).

(38) Lukin, O., Godt, A. \& Vogtle, F. Residual topological isomerism of intertwined molecules. Chemistry 10, 1878-1883 (2004).

(39) Martinez-Cuezva, A., Saura-Sanmartin, A., Alajarin, M. \& Berna, J. Mechanically Interlocked Catalysts for Asymmetric Synthesis. ACS Catal. 10, 7719-7733 (2020).

(40) Pairault, N. \& Niemeyer, J. Chiral Mechanically Interlocked Molecules - Applications of Rotaxanes, Catenanes and Molecular Knots in Stereoselective Chemosensing and Catalysis. Synlett 29, 689-698 (2018).

(41) Mitra, R., Zhu, H., Grimme, S. \& Niemeyer, J. Functional Mechanically Interlocked Molecules: Asymmetric Organocatalysis with a Catenated Bifunctional Bronsted Acid. Angew. Chem. Int. Ed. 56, 11456-11459 (2017).

(42) Pairault, N. et al. Heterobifunctional Rotaxanes for Asymmetric Catalysis. Angew. Chem. Int. Ed. 59, 5102-5107 (2020).

(43) Dommaschk, M., Echavarren, J., Leigh, D. A., Marcos, V. \& Singleton, T. A. Dynamic Control of Chiral Space Through Local Symmetry Breaking in a Rotaxane Organocatalyst. Angew. Chem. Int. Ed. 58, 14955-14958 (2019).

(44) Heard, A. W. \& Goldup, S. M. Synthesis of a Mechanically Planar Chiral Rotaxane Ligand for Enantioselective Catalysis. Chem 6, 994-1006 (2020). 
(45) Gaedke, M. et al. Chiroptical inversion of a planar chiral redox-switchable rotaxane. Chem. Sci. 10, 10003-10009 (2019).

(46) Corra, S. et al. Chemical On/Off Switching of Mechanically Planar Chirality and Chiral Anion Recognition in a [2]Rotaxane Molecular Shuttle. J. Am. Chem. Soc. 141, 9129-9133 (2019).

(47) Wood, C. S., Ronson, T. K., Belenguer, A. M., Holstein, J. J. \& Nitschke, J. R. Two-stage directed self-assembly of a cyclic [3]catenane. Nat Chem 7, 354-358 (2015).

(48) Caprice, K. et al. Diastereoselective Amplification of a Mechanically Chiral [2]Catenane. J Am Chem Soc 143, 11957-11962 (2021).

(49) Cui, Z., Lu, Y., Gao, X., Feng, H. J. \& Jin, G. X. Stereoselective Synthesis of a Topologically Chiral Solomon Link. J Am Chem Soc 142, 13667-13671 (2020).

(50) Carpenter, J. P. et al. Controlling the shape and chirality of an eight-crossing molecular knot. Chem 7, 1534-1543 (2021).

(51) Leigh, D. A. et al. Tying different knots in a molecular strand. Nature 584, 562-568 (2020).

(52) David, A. H. G. \& Stoddart, J. F. Chiroptical Properties of Mechanically Interlocked Molecules. Israel Journal of Chemistry 61, 608-621 (2021). 